\title{
Evaluating the Efficacy of Remifentanil-Propofol versus Isoflurane in Reducing Blood Loss with Considering Depth of Anesthesia during Endoscopic Sinus Surgery*
}

\author{
Mohammad Ali Haghbin ${ }^{1}$, Hossein Hakimzadeh ${ }^{1}$, Mohammad Shabani2 ${ }^{2 \#}$ \\ ${ }^{1}$ Department of Anesthesiology, Kerman University of Medical Sciences, Kerman, Iran; ${ }^{2}$ Kerman Neuroscience Research Center, \\ Kerman University of Medical Sciences, Kerman, Iran. \\ Email: "shabanimoh@yahoo.com
}

Received February $26^{\text {th }}, 2013$; revised March $27^{\text {th }}, 2013$; accepted April $20^{\text {th }}, 2013$

Copyright (C) 2013 Mohammad Ali Haghbin et al. This is an open access article distributed under the Creative Commons Attribution License, which permits unrestricted use, distribution, and reproduction in any medium, provided the original work is properly cited.

\begin{abstract}
Several methods have been suggested to decrease bleeding during surgery, one of which is the usage of hypotensive anesthetic agents. Endoscopic Sinus Surgery (ESS) is one of the surgeries which need a clear field and the amount of bleeding profoundly impacts the ability of surgeons. Current study was designed to evaluate the effect of remifentanil combined with propofol versus Isoflurane on blood loss during ESS and its possible interaction with Depth of Anesthesia. Fifty one patients comprising 15 females and 36 males undergoing ESS were chosen for this study. One group received propofol and remifentanil to induce and maintain anesthesia and the second group received Isoflurane. Mean Arterial Blood Pressure (MAP), Depth of Anesthesia and total blood loss were recorded for the patients of both group. Data were analyzed using t-test, Repeated Measures ANOVA and Pearson's correlation. $\mathrm{P}<0.05$ was considered statistically significant. Both agents induced hypotension during surgery. No significant difference was observed in total blood loss between two groups. DA was decreased in both groups, but no significant correlation was found between DA and MAP or DA and blood loss. There is no significant difference between remifentanil combined with propofol and Isoflurane in decreasing blood loss during ESS, thus we suggest usage of hypotensive anesthetic agent regardless of its type is ESS.
\end{abstract}

Keywords: Isoflurane; Propofol; Remifentanil; Blood Loss; Endoscopic Sinus Surgery

\section{Introduction}

Having a clear visual field is a critical part of a good surgery. Endoscopic Sinus Surgery (ESS), like other surgeries need a clean and blood-free field and due to the nature of the organs which are operated, even a drop of blood can disturb the field for the surgeon and lead to an increased surgery time and the operation becomes more difficult [1].

Several maneuvers have been recruited to improve the visual field and give a better visualization to the surgeon. Using hypotensive agents during surgery has been suggested to be effective to provide a good visual field for the surgeons. Several agents such as alpha2A adrenergic agonists (clonidine and dexmedotomidine), vasodilators (nitroprusside, nicardipine and nitroglycerine), beta adrenergic antagonists (propranolol and esmolol) and

*Conflict of Interest: The authors declare no conflict of interest.

${ }^{\#}$ Corresponding author. high doses of inhaled anesthetics (Isoflurane and sevoflurane) have been used to provide a hypotensive condition during surgery [2-4].

Efficacy of Isoflurane (Iso) versus remifentanil combined with propofol (RPP) in reducing blood loss during ESS has been shown before propofol administration reduced bleeding in a more extent compared to Isoflurane [5]. In another study, effect of sevoflurane, desflurane and remifentanil combined with propofol on surgical conditions of patients undergoing ESS was evaluated by Yoo et al. They showed that there is no difference between these three anesthetic agents in reducing Blood Pressure (BP) and Heart Rate (HR) [1].

Contradictory results have been shown regarding efficacy of remifentanil combined with propofol and other hypotensive agents in reducing blood loss during ESS, and to our knowledge, no study has addressed the efficacy of RPP versus Iso in reducing blood loss during 
EES. We also hypothesized that Depth of Anesthesia might have a role in hypotension and MAP; therefore we performed this prospective randomized study on 51 patients undergoing ESS in Shafa hospital, Kerman, Iran.

\section{Methods and Materials}

\subsection{Subject}

Fifty one patients undergoing ESS were enrolled in this randomized prospective trial. Subjects were 36 males with median age of $34.44 \pm 11.53$ and 15 females with median age of $48.4 \pm 12.87$. Ethical committee approval was obtained from Kerman University of Medical Sciences [Code: EC/KNRC/90-69]. Exclusion criteria for this study was: history of allergic reaction to the anesthetic agents used, patients with history of hypertension, patients receiving treatment with anti-hypertension drugs, patients receiving anti-coagulant drugs, patients with a history of cardiovascular, hepatic or renal disorder, opioid and analgesics abuse, chronic treatment with calcium channel blockers and patients with diabetes. Disease intensity was evaluated by CT scan graphing and patients with the same surgical difficulty level were chosen for this study. Patients were randomly allocated to the RPP or Iso groups. All the participants were informed about the objective of the study and signed the informed consent, but they were kept blind about the anesthetic agent used while the surgeon was aware of the anesthetic agent used.

Patients were pretreated with $1 \mathrm{mg} / \mathrm{kg}$ midazolam and $1 \mu \mathrm{g} / \mathrm{kg}$ fentanyl. Electrocardiography (EKG), pulse oximetry, body temperature, noninvasive Blood Pressure and Bispecteral Index (BIS) [6] were monitored (Bispectral index is one of several technologies which purport to monitor Depth of Anesthesia). The target RPP and Iso were set by entering the patients age and weight into the TCI unit. Patients were ventilated with $100 \%$ oxygen during induction. Induction of anesthesia was performed by $1.5 \mathrm{mg} / \mathrm{kg}$ propofol and $0.15 \mathrm{mg} / \mathrm{kg}$ cis-atracurium to paralyze muscles and loss of the eyelash reflex and verbal response were considered as anethetic criterion. The lungs were mechanically ventilated along with the RPP and Iso maintenance target infusions; RPP was infused with $150 \mu \mathrm{g} / \mathrm{kg} / \mathrm{min}$ propofol and $25 \mu \mathrm{g} / \mathrm{kg} / \mathrm{min} \mathrm{Re}-$ mifentanil. Volatile anesthetic Isoflurane (Minimum Alveolar Concentration MAC 1.2) with $50 \% \mathrm{~N}_{2} \mathrm{O}+50 \%$ $\mathrm{O}_{2}$ ) were given to maintain an adequate Depth of Anesthesia, as judged by clinical signs and hemodynamic responses to surgical stimuli. In duration of study, MAP, pulse oximetry, body temperature was monitored by noninvasive Electrocardiography monitor that is including EKG, thermometer, indicator and pulse oximeter.
At the end of each surgery, blood was collected from each suction canister which included a known amount of heparin. $\mathrm{Hb}$ concentration of blood collected in canister and patient's blood sample was measured using cyanmethemoglobin method and inserted into the following formula to calculate the total shed blood [7].

Total hemoglobin in shed blood $=(\mathrm{Hg}$ in canister of suction) $(\mathrm{g} / \mathrm{ml}) *$ (volume of canister) $(\mathrm{mL})$.

Volume of shed blood $=($ total hemoglobin in shed blood) $(\mathrm{g}) /($ mean plasma $\mathrm{Hb}$ concentration $)(\mathrm{g} / \mathrm{mL})$.

Data were analyzed using SPSS v.16. To compare blood loss between RPP and Iso group, student's t-test was used and for comparison of Depth of Anesthesia (DA) and Mean Arterial Pressure (MAP) during different intervals after initiation of surgery, we used repeated measures ANOVA and between subject's analysis to compare DA and MAP in different intervals between Iso and RPP group. To evaluate the possible correlation between DA and MAP, we used pearson's correlation test. $\mathrm{P}<0.05$ was considered statistically significant. Data are presented as mean \pm S.E.M.

\section{Results}

Shed blood was calculated by the formula mentioned above. Mean blood loss for the RPP group was $125.95 \pm$ $18.3 \mathrm{~mL}$ and for the Iso group, it was $136.81 \pm 17.6 \mathrm{~mL}$. The difference between RPP and Iso group was not statistically significant ( $\mathrm{t}$-test, $\mathrm{t}=-0.426, \mathrm{P}=0.67$ ).

MAP was significantly reduced in groups compared to baseline measurement (repeated measures ANOVA), showing hypotension, but the difference between Iso and RPP was not meaningful (between subject analysis) (Figure 1).

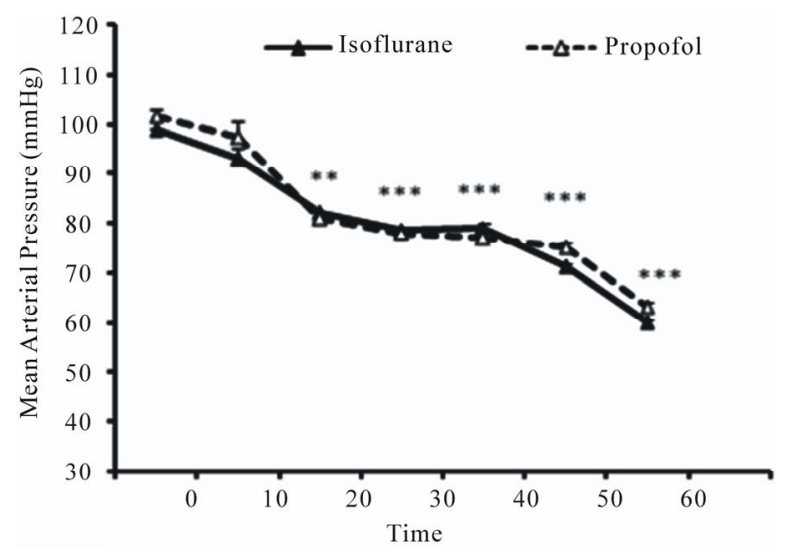

Figure 1. Mean Arterial Pressure (MAP) between propofol and Isoflurane group. Both agents induced hypotension compared to baseline, but no significant difference was observed between two drugs ( ${ }^{*} \mathrm{P}<0.05$ repeated measures ANOVA, between subject analysis. Data presented as mean \pm S.E.M.). 
DA was also significantly reduced in both groups compared to baseline, but interestingly, the difference between two groups was meaningful from minute 30 to minute 70 of surgery (Figure 2).

Possible correlation between MAP and DA was tested using Pearson correlation. Results are shown in Table 1. there was no meaningful correlation between DA and MAP in different periods after initiation of surgery except for the 50 - 60 minutes after initiation of surgery interval which a fair negative correlation was found between these two variables in PRR group $(\mathrm{P}=0.025, \mathrm{R}=$ -0.476 ) (Table 1). There were no significant differences between two groups in body temperature and $\mathrm{O}_{2}$ saturation (data not shown).

\section{Discussion}

In this study, we evaluated the effect of RPP and Isoflurane on blood loss during EES and the possible effect of

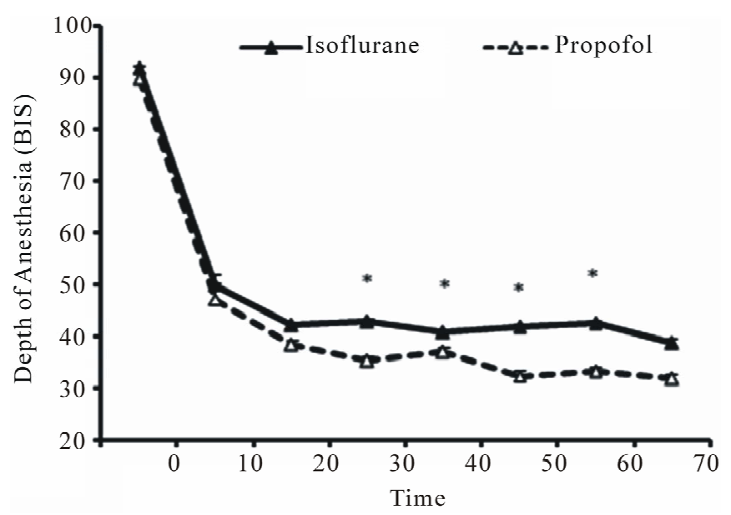

Figure 2. Depth of Anesthesia (DA) compared within subjects in each group and between propofol and Isoflurane group. The difference between two groups was meaningful from minute 30 to minute 70 post surgery $\left({ }^{*} P<0.05\right.$ repated measure ANOVA. Data presented as mean \pm S.E.M.).

Table 1. Correlation between Mean Arterial Pressure (MAP) and Depth of Aesthesia (DA) was tested using pearson's correlation in different time points from initiation of surgery. No significant correlation was detected between these two variables (Pearson's correlation, ${ }^{*} \mathbf{P}<\mathbf{0 . 0 5}$ ).

\begin{tabular}{ccc}
\hline Time points & Propofol & Isoflurane \\
\hline DA0, MAP0 & $\mathrm{P}>0.05$ & $\mathrm{P}>0.05$ \\
DA10, MAP10 & $\mathrm{P}>0.05$ & $\mathrm{P}>0.05$ \\
DA20, MAP20 & $\mathrm{P}>0.05$ & $\mathrm{P}>0.05$ \\
DA30, MAP30 & $\mathrm{P}>0.05$ & $\mathrm{P}>0.05$ \\
DA40, MAP40 & $\mathrm{P}>0.05$ & $\mathrm{P}>0.05$ \\
DA50, MAP50 & $\mathrm{R}=-0.476(\mathrm{P}=0.025)$ & $\mathrm{P}>0.05$ \\
DA60, MAP60 & $\mathrm{P}>0.05$ & $\mathrm{P}>0.05$ \\
DA70, MAP70 & $\mathrm{P}>0.05$ & $\mathrm{P}>0.05$ \\
\hline
\end{tabular}

Depth of Anesthesia (DA) on Blood Pressure. We found no significant difference in shed blood collected during surgery between two groups and no significant correlation was found between Blood Pressure and DA in different intervals after initiation of surgery.

Nature of surgeries dictates the usage of better maneuvers and approaches to achieve a better visual field. Several maneuvers have been proposed to improve the visual field and one of them is using hypotensive agents to reduce blood loss during surgery. Propofol combined with remifentanil is used as a hypotensive anesthetic agent, and some studies have evaluated the efficacy of this agent in reducing blood loss during surgery.

Ryu et al. evaluated the efficacy of remifentanil versus magnesium sulphate in middle ear surgery and there was no significant difference in surgical conditions during and after surgery [8]. Although we did not evaluate the surgical conditions in our study, but the hypotension observed in RRP and Iso group is similar to Ryu study, which proposes RPP as a good option for hypotension. In our study, we did not evaluate the surgical condition and the field clarity, which is a limitation to this study.

Blackwell and colleagues evaluated the efficacy of propofal versus Isoflurane on reducing blood loss during EES; they showed that propofol usage results in less blood loss during surgery, which is inconsistent with our study [9]. One possible explanation for this difference might be the age difference in their study, Isoflurane group patients were 15 years older than the Propofol group and this difference might not justify propofol usage for reducing blood loss during surgery, along with lower sample size compared to our study. One other explanation might be due to different level of EES difficulty, as shown by Ahn et al. (2007).

Ahn et al. (2007) showed that in patients with low grade of EES difficulty, there is no difference between RPP and Desuflurane group but in patients with high grade of EES difficulty, propofol was better at providing a better surgical field and lesser blood loss during surgery [10].

Our results are consistent with Ankichetty et al. (2011) and Pavlin et al. study (1999), as they have also demonstrated that propofol has no advantage versus Isoflurane in reducing blood loss $[5,9,11]$. Although Pavlin et al. showed that propofol provides a better surgical condition compared to Isoflurane and also propofol resulted in a lesser time of hospitalization, one thing that we did not evaluate in our study [5].

In one study recently published by Yoo et al. (2010), the efficacy of RPP, Iso and Desflurane on surgical condition was evaluated and consistent with our findings, no difference in Blood Pressure was observed among these three anesthetic agents [1]. Yoo et al. also evaluated the 
surgical grade scores for these three agents and no difference was found among them, something that we did not evaluate in our study.

One hypothesis we tested in our study is the possible role of DA in MAP. Correlation tests did not reveal any significant relationship between RPP and Iso, except for 50 - 60 min interval, which a significant negative correlation was found. As expected, BIS index was significantly reduced in both groups compared to baseline, showing increased DA. These findings deny a strong relationship between DA and MAP in different intervals after initiation of surgery.

As a conclusion, we have shown that RPP and Iso have no advantages over each other in reduction of blood loss during ESS. Although both agents are hypotensive, there is no prominent evidence for preference of anesthetic agent which would improve surgical conditions and blood loss during ESS.

\section{Acknowledgements}

The present manuscript is the product of a research project that was approved by the Kerman University of Medical Science.

IRB: This study was approved by the Iranian Registery of Clinical Trials [Code: IRCT201202067488N3] and registered with the European Clinical Trials Database. (http://www.irct.ir/user.php?nav=homepage\&lan=en\&nu mber $=$ ).

\section{REFERENCES}

[1] H. S. Yoo, J. H. Han, S. W. Park and K. S. Kim, "Comparison of Surgical Condition in Endoscopic Sinus Surgery Using Remifentanil Combined with Propofol, Sevoflurane, or Desflurane," Korean Journal of Anesthesiology, Vol. 59, No. 6, 2010, pp. 377-382. doi:10.4097/kjae.2010.59.6.377

[2] C. S. Degoute, M. J. Ray, P. Y. Gueugniaud and C. Dubreuil, "Remifentanil Induces Consistent and Sustained Controlled Hypotension in Children during Middle Ear Surgery," Canadian Journal of Anesthesia, Vol. 50, No. 3, 2003, pp. 270-276. doi:10.1007/BF03017797

[3] C. S. Degoute, M. J. Ray, M. Manchon, C. Dubreuil and V. Banssillon, "Remifentanil and Controlled Hypotension; Comparison with Nitroprusside or Esmolol during Tym- panoplasty," Canadian Journal of Anesthesia, Vol. 48, No. 1, 2001, pp. 20-27. doi:10.1007/BF03019809

[4] F. Richa, et al., "Comparison between Dexmedetomidine and Remifentanil for Controlled Hypotension during Tympanoplasty," European Journal of Anaesthesiology, Vol. 25, No. 5, 2008, pp. 369-374. doi:10.1017/S0265021508003761

[5] J. D. Pavlin, P. S. Colley, E. A. Weymuller, G. V. Norman, H. C. Gunn and M. E. Koerschgen, "Propofol versus Isoflurane for Endoscopic Sinus Surgery," American Journal of Otolaryngology, Vol. 20, No. 2, 1999, pp. 96101. doi:10.1016/S0196-0709(99)90018-2

[6] M. Nakayama, H. Ichinose, S. Yamamoto, N. Kanaya and A. Namiki, "The Bispectral Index Response to Tracheal Intubation Is Similar in Normotensive and Hypertensive patients," Canadian Journal of Anesthesia, Vol. 49, No. 5, 2002, pp. 458-460. doi:10.1007/BF03017920

[7] A. G. Beule, F. Wilhelmi, T. S. Kuhnel, E. Hansen, K. J. Lackner and W. Hosemann, "Propofol versus Sevoflurane: Bleeding in Endoscopic Sinus Surgery," Otolaryngol Head Neck Surgery, Vol. 136, No. 1, 2007, pp. 45-50. doi:10.1016/j.otohns.2006.08.006

[8] J. H. Ryu, I. S. Sohn and S. H. Do, "Controlled Hypotension for Middle Ear Surgery: A Comparison between Remifentanil and Magnesium Sulphate," British Journal of Anaesthesia, Vol. 103, No. 4, 2009, pp. 490-495. doi:10.1093/bja/aep229

[9] K. E. Blackwell, D. A. Ross, P. Kapur and T. C. Calcaterra, "Propofol for Maintenance of General Anesthesia: A Technique to Limit Blood Loss during Endoscopic Sinus Surgery," American Journal of Otolaryngology, Vol. 14, No. 4, 1993, pp. 262-266. doi:10.1016/0196-0709(93)90072-F

[10] H. Ahn, S. K. Chung, H. J. Dhong, H. Y. Kim, J. H. Ahn, S. M. Lee, T. S. Hahm and J. K. Kim, "Comparison of Surgical Conditions during Propofol or Sevoflurane Anaesthesia for Endoscopic Sinus Surgery," British journal of Anaesthesia, Vol. 100, No. 1, 2008, pp. 50-54. doi:10.1093/bja/aem304

[11] S. P. Ankichetty, M. Ponniah, V. Cherian, S. Thomas, K. Kumar, L. Jeslin, K. Jeyasheela and N. Malhotra, "Comparison of Total Intravenous Anesthesia Using Propofol and Inhalational Anesthesia Using Isoflurane for Controlled Hypotension in Functional Endoscopic Sinus Surgery," Journal of Anaesthesiology Clinical Pharmacology, Vol. 27, No. 3, 2011, pp. 328-332. doi: $10.4103 / 0970-9185.83675$ 